\title{
Noncooperative Power Control and Transmission Scheduling in Wireless Collision Channels
}

\author{
Paper ID: 1569083916 - 10 pages
}

\begin{abstract}
We consider a wireless collision channel, shared by a finite number of mobile users who transmit to a common base station using a random access protocol. Mobiles are selfoptimizing, and wish to minimize their individual average power investment subject to minimum-throughput demand. The channel state between each mobile and the base station is stochastically time-varying and is observed by the mobile prior to transmission. Given the current channel state, a mobile may decide whether to transmit or not, and to determine the transmission power in case of transmission. In this paper, we investigate the properties of the Nash equilibrium of the resulting game in multiuser networks.

We characterize the best-response strategy of the mobile and show that it leads to a "water-filling"-like power allocation. Our equilibrium analysis then reveals that one of the possible equilibria is uniformly best for all mobiles. Furthermore, this equilibrium can be reached by a simple distributed mechanism that does not require specific information on other mobiles' actions. We then explore some additional characteristics of the distributed power control framework. Braess-like paradoxes are reported, where the use of multiple power levels can diminish system capacity and also lead to larger per-user power consumption, compared to the case where a single level only is permitted.
\end{abstract}

\section{INTRODUCTION}

\subsection{Background and Motivation}

Wireless technologies are broadly used nowadays for both data and voice communications. The transmission protocols of wireless devices need to cope with the scarce resources available, such as bandwidth and energy. Additional difficulties relate to the dynamic nature of wireless networks. For example, the mobility of terminals and the frequent change in their population introduces new challenges for routing protocols. An additional distinctive dynamic feature of wireless communications is the possible frequent time variation in the channel quality between the sender and the receiver, an effect known as channel fading [1].

Motivated by scalability considerations, it has been advocated in recent years that mobiles should have the freedom to distributively adjust their transmission parameters (e.g., [2]). Perhaps the most effective parameter that can be autonomously controlled is the transmission power. Power control becomes extremely significant when the mobile is aware of its channel quality. Evidently, if channel state information (CSI) is available, it can be exploited for increased rates and better usage of the available power $[1,3,4]$.

Much research has accordingly been devoted to study the optimal power allocation in face of varying channel conditions. It turns out that the single-user closed-form solution to this problem is analogous to "water-filling" (or water-pouring), in the sense that transmissions are kept for better channel quality (see [5]). In shared, multiuser, wireless domains this power-control framework becomes more involved, as the performance of each mobile is significantly affected by the actions taken by other mobiles. A classic model, which we consider in this work, is that of a collision channel, where multiple simultaneous transmissions lead to the loss of all transmitted data.

In considering user-controlled transmissions, two basic (and commensurate) requirements should be considered:

(i) Distributed control: Each user should base its decision on locally available information (perhaps augmented with some centrally controlled data which is easily available to all).

(ii) Self-optimizing framework: If tight central regulation is to be avoided, the procedures used by each mobile should be perceived efficient from its own point of view.

Our work considers the combined power-control and transmission scheduling problem in a time-slotted collision channel. At every time slot each user may observe its own channel quality, and decide whether to transmit or not, and if so at which power level (chosen from a discrete set). Our focus in this paper is on local stationary transmission strategies, in which this decision can depend (only) on the current channel state of the mobile. A basic assumption of our user model is that each mobile user has some throughput requirement, which it wishes to sustain with a minimal power investment. 
The required throughput of each user may be dictated by its application (such as video or voice which may require fixed bandwidth), or mandated by the system. Users cannot coordinate their transmissions, and adjust their transmission decisions to minimize their power investment based on network conditions. This situation is naturally modeled and analyzed as a noncooperative game between the mobiles. The basic objective of this paper is to obtain structural properties for the associated Nash equilibria, and investigate the effects of the physical parameters (e.g., the channel state distribution, the available power levels) on equilibrium performance.

The technological relevance for our work lies, for example, in Wireless Personal and Local Area Network systems (WPANs, WLANs), where the underlying network users may have diverse (application-dependent) throughput requirements. The leading WLAN standard 802.11x [6], employs a random access protocol, whose principles are based on the Aloha protocol [7]. Interestingly, on-going IEEE standardization activity (the $802.11 \mathrm{n}$ standard) focuses on the incorporation of CSI for better network utilization. This last fact motivates our research, as it can contribute to the understanding and improvement of present and future wireless platforms.

\subsection{Related Work}

Significant research efforts have been dedicated over the last decade to the study of wireless networks through gametheoretic tools. In particular, several papers (e.g., $[8,9,10])$ have studied the equilibrium properties of power allocation games, where mobile users adjust their transmission power to meet some objective (such as maximizing the throughput, or the energy investment per bit). The above papers consider a static setup, where mobiles adjust their power based on average network conditions. Consequently, an equilibrium is characterized by fixed transmission powers for all users.

Recently, [11, 12] consider the power allocation game under time-varying channel conditions, which require users to adjust their transmission power as a function of the channel state. In [11] it is assumed that the channel state of a particular user is available to all others, an assumption that might be hard to justify in practice. As in our model, [12] considers the case where only private channel state information is available. The reception model that is studied in the above references supports multipacket-reception, as opposed to the collision channel studied here. The user objective in $[11,12]$ assumes that users maximize individual throughput subject to power constraints. In addition, these two papers study a specific rate function that corresponds to the Gaussian channel.

In [13], a similar model is considered, except that a single power level only is available to each user. We extend this work by allowing users to control their transmission power too. For the single power case, it was established in [13] that two equilibria exist, where the better one coincides with the optimal (centralized assigned) stationary transmission strategy. In our extended model we show that multiple equilibria may exist (not necessarily two), where one is best for all users, yet can be inferior to the optimal solution.

\subsection{Contribution and Paper Organization}

This paper presents a study of the non-cooperative power control game between the channel-aware, self-interested network users. The main contributions are summarized below.

- We provide a general model for the distributed power control and transmission scheduling problem, that incorporates stochastic channel variation, with which the interaction of selfish users may be studied.

- We characterize the best-response transmission strategy for each mobile, i.e., the individually optimal power control, given that other user strategies are fixed. The novelty here is in considering the discrete water-filling problem, where the channel quality takes a finite number of values and where the number of power levels available to each mobile is finite as well. This framework is more realistic than the continuous case studied in classical water-filling literature $([1,5]$ and references therein).

- Based on the structural properties of each user's best response, we not only characterize each equilibrium in isolation, but are also able to obtain important system-wide properties. We show that equilibria are completely ordered in terms of the per-user power investment; hence, there is a power-efficient equilibrium, which is best for all. This equilibrium can be reached through best response dynamics that requires minimal information structure for each mobile.

- On the negative side, we show that the power-efficient equilibrium is usually inferior to the centrally assigned power schedule. In addition, we demonstrate that the freedom given to users in the form of multiple power levels, might have negative effects on network performance, in terms of both channel capacity and overall power consumption. This fact should be therefore considered in the design of present and future medium access protocols.

We emphasize that the above results are valid under general assumptions on the channel state distribution, the allowed power-levels and the associated rates. Moreover, we make no symmetry assumptions on the users, which are imposed in much of the existing work in this area.

The structure of the paper is as follows. We first present the general model (Section 2), and define the Nash equilibrium point of the noncooperative game. Structural results of the Nash equilibria are derived in Section 3. In addition, we show that if the required rates are feasible, there exists an equilibrium point which is uniformly best for all users in terms of the power investment. Accordingly, in Section 4 we suggest a simple distributed mechanism that converges to that equilibrium. In Section 5 we address the efficiency loss incurred by selfish user behavior, and identify a couple of Braess-like paradoxes. Section 6 discusses some consequences of our results. Conclusion and further research direction are drawn in Section 7.

\section{THE MODEL}

We consider a wireless network, shared by a finite set of mobile users $\mathcal{M}=\{1, \ldots, M\}$ who transmit to a common base station over a shared collision channel. Time is slotted, so that each transmission attempt takes place within slot 
boundaries that are common to all. A finite set of power levels ${ }^{1} \mathcal{Q}^{m}=\left\{Q_{0}^{m}, Q_{1}^{m}, \ldots, Q_{J^{m}}^{m}\right\}$ are available to each mobile $m$, where $0=Q_{0}^{m}<Q_{1}^{m}, \cdots<Q_{J m}^{m}$. A transmission at any (positive) power level is successful only if no other user attempts transmission simultaneously. Thus, at each time slot, at most one user can successfully transmit to the base station.

To further specify our model, we start with a description of the channel between each user and the base station (Section 2.1), ignoring the possibility of collisions. In Section 2.2 , we formalize the user objective and formulate the noncooperative game which arises in a multi-user shared network.

\subsection{The Single-User Channel}

Our model for the channel between each user and the base station is characterized by two basic quantities.

a. Channel State. We assume that the channel state (or quality) between mobile $m$ and the base station evolves as an ergodic Markov chain $X^{m}(t)$ taking values in a finite set $\mathcal{X}^{m}=\left(x_{1}^{m}, x_{2}^{m}, \ldots, x_{I^{m}}^{m}\right)$ of $I^{m}$ states. For convenience we shall assume that the states are ordered from worst $\left(x_{1}^{m}\right)$ to best $\left(x_{I^{m}}^{m}\right)$ and denote this relation by $x_{1}^{m}<x_{2}^{m}<\ldots<$ $x_{I^{m}}^{m}$. The Markov chains $X^{m}(t), m=1 \ldots M$, are assumed to be independent. We denote by $\pi^{m}$ the row vector of steady state probabilities of the Markov chain $X^{m}(t)$, and by $\pi_{i}^{m}$ its $i$ th entry corresponding to state $x_{i}^{m} \in \mathcal{X}^{m}$.

b. Expected data rate. Let $R_{i, j}^{m} \geq 0$ denote the expected data rate (in bits per second) that user $m$ can sustain at a given slot as a function of the current channel state $x_{i}^{m}$ and the power level $Q_{j}^{m}$ assigned for the transmission. We assume that the data rate strictly increases with the channel quality, and further that it strictly increases with the transmission power. That is,

$$
\begin{array}{ll}
R_{1, j}^{m}<R_{2, j}^{m}<\cdots<R_{I^{m}, j}^{m}, & j \in\left\{1, \ldots J^{m}\right\} \\
R_{i, 1}^{m}<R_{i, 2}^{m}<\cdots<R_{i, J^{m}}^{m}, & i \in\left\{1, \ldots I^{m}\right\} ;
\end{array}
$$

naturally, $R_{i, 0}^{m}=0$, since $Q_{0}^{m}=0$ by definition.

Example: Assume that white Gaussian noise with a spectral density of $N_{0} / 2$ is added to the transmitted signal. Then if a user can optimize its coding scheme to approach the Shannon capacity, the average rate that can be sustained is given by the following rate function

$$
R_{i, j}^{m}=\log \left(1+x_{i}^{m} Q_{j}^{m} / N_{0}\right) .
$$

The Gaussian channel case is broadly studied in the Information Theory literature. We shall consider the rate function (3) in our experiments.

\subsection{User Objective and Game Formulation}

In this subsection we describe the user objective and the non-cooperative game which arises as a consequence of the user interaction over the collision channel. We characterize stationary transmission strategies, which are central in this

${ }^{1} \mathrm{~A}$ continuum of power levels can be treated as well, and in fact turns out to be analytically simpler. Here we choose to focus on the finite case which is more realistic. paper, and then define the Nash equilibrium of the game within this class of strategies.

\subsubsection{Basic Definitions}

We associate with each user $m$ a throughput demand $\rho^{m}$ (in bits per second) which it wishes to deliver over the network. The objective of each user is to minimize its average transmission power while maintaining the effective data rate at (or above) this user's throughput demand. We further assume that users always have packets to send, yet they may delay transmission to a later slot to accommodate their required throughput with minimal power investment.

Our focus in this paper is on local and stationary transmission strategies, in which the transmission power decision (which includes the decision not to transmit at all) can depend only on the current state of the mobile $x_{i}^{m}$. The user does not have any information regarding the channel state of other users. For any given channel state, the mobile decision may include randomization over the available powers. A formal definition is provided below.

Definition 2.1 (LOCAlly Stationary Strategy). $A$ stationary strategy for user $m$ is represented by an $I^{m} \times$ $\left(J^{m}+1\right)$ matrix $\mathbf{q}^{m}$, where its $(i, j)$ entry, denoted $q_{i, j}^{m}$, corresponds to the probability that user $m$ will transmit at power $Q_{j}^{m}$ when the observed channel state is $x_{i}^{m}$. As such, the set of feasible stationary strategies is given by

$$
\Delta^{m}=\left\{q_{i, j}^{m} \geq 0, \quad \sum_{j=0}^{J^{m}} q_{i, j}^{m}=1 \forall i=1, \ldots I^{m}\right\} .
$$

For simplicity, we shall refer to the above defined strategy as stationary strategy. We also define two user-specific quantities that are derived from a given stationary strategy $\mathbf{q}^{m}$.

- The transmission probability $p^{m}\left(\mathbf{q}^{m}\right)$ in a slot, given by

$$
p^{m}\left(\mathbf{q}^{m}\right)=\sum_{i=1}^{I^{m}} \pi_{i}^{m}\left(\sum_{j=1}^{J^{M}} q_{i, j}^{m}\right) .
$$

- The collision-free data-rate $H^{m}\left(\mathbf{q}^{m}\right)$, which stands for the average data-rate of successful transmissions, namely

$$
H^{m}\left(\mathbf{q}^{m}\right)=\sum_{i=1}^{I^{m}} \pi_{i}^{m}\left(\sum_{j=1}^{J^{M}} q_{i, j}^{m} R_{i, j}^{m}\right) .
$$

We use the term multi-strategy when referring to a collection of (stationary) user strategies, and denote it by $\mathbf{q} \triangleq$ $\left(\mathbf{q}^{1}, \ldots, \mathbf{q}^{M}\right)$. The notation $\mathbf{q}^{-m}$ will be used for the transmission strategies of all users but for the $m$-th one. Note that the probability that no user from the set $\mathcal{M} \backslash\{m\}$ transmits in a given slot is given by $\prod_{l \neq m}\left(1-p^{m}\left(\mathbf{q}^{l}\right)\right)$. Since the transmission decision of each user is independent of the decisions of other users, the expected data rate of user $i$, denoted $r^{m}(\mathbf{q})$, is given by

$$
r^{m}(\mathbf{q})=H^{m}\left(\mathbf{q}^{m}\right) \prod_{l \neq m}\left(1-p^{l}\left(\mathbf{q}^{l}\right)\right) .
$$


The basic assumption of our model is that users are selfoptimizing and are free to determine their own transmission strategy in order to fulfill their objectives. Furthermore, users are unable to coordinate their transmission decisions. This situation is modeled and analyzed in our paper as a non-cooperative game [14] between the $M$ users. In particular, we are interested in the Nash equilibrium point of the game.

A Nash equilibrium point (NEP) for our model is a multistrategy $\mathbf{q}=\left(\mathbf{q}^{1}, \ldots, \mathbf{q}^{M}\right)$, which is self-sustaining in the sense that all throughput constraints are met, and neither user can lower its power investment by unilaterally modifying its transmission strategy. Formally,

Definition 2.2 (NASH EQUILIBRIUM POINT). A multistrategy $\mathbf{q}=\left(\mathbf{q}^{1}, \ldots, \mathbf{q}^{M}\right)$ is a Nash equilibrium point if for every $m \in \mathcal{M}$,

$\mathbf{q}^{m} \in \underset{\tilde{\mathbf{q}}^{m} \in \Delta^{m}}{\operatorname{argmin}}\left\{\sum_{i=1}^{I^{m}} \pi_{i}^{m} \sum_{j=1}^{J^{M}} \tilde{q}_{i, j}^{m} Q_{j}^{m}: r^{m}\left(\tilde{\mathbf{q}}^{m}, \mathbf{q}^{-m}\right) \geq \rho^{m}\right\}$.

Using game-theoretic terminology, a Nash equilibrium is a multi-strategy $\mathbf{q}=\left(\mathbf{q}^{1}, \ldots, \mathbf{q}^{M}\right)$ so that each $\mathbf{q}^{m}$ is a best response of user $m$ to $\mathbf{q}^{-m}$, in the sense that the user's cost (average power in our case) is minimized.

Noting (6), it is important to emphasize that the only interaction between users is through the effective collision rate over the shared channel. This observation is significant in the context of the game dynamics, as the only external information that is required for best response adaptation is not user-specific and can be relatively easily measured.

REMARK 1. As noted above, we restrict attention here to stationary strategies. When the channel state process is i.i.d., it may be shown that a Nash equilibrium in locally stationary strategies is in fact a Nash equilibrium in general strategies. For more general state processes (e.g., Markovian), this need not be the case and the restriction to stationary strategies is upheld for simplicity of implementation.

\section{EQUILIBRIUM ANALYSIS}

In this section we characterize the Nash equilibria (7) of the network under stationary transmission strategies. Our analysis starts by examining the best-response strategy of each mobile. In particular, we demonstrate that classic "water-filling" properties for optimal power control carry over to the noncooperative-game framework. We then show that one of the possible equilibrium points is best for all users in terms of their power investment.

\subsection{Basic Properties}

This subsection provides some basic properties of the bestresponse strategy. Our first result states that the throughput demands should be met with equality at every equilibrium point.

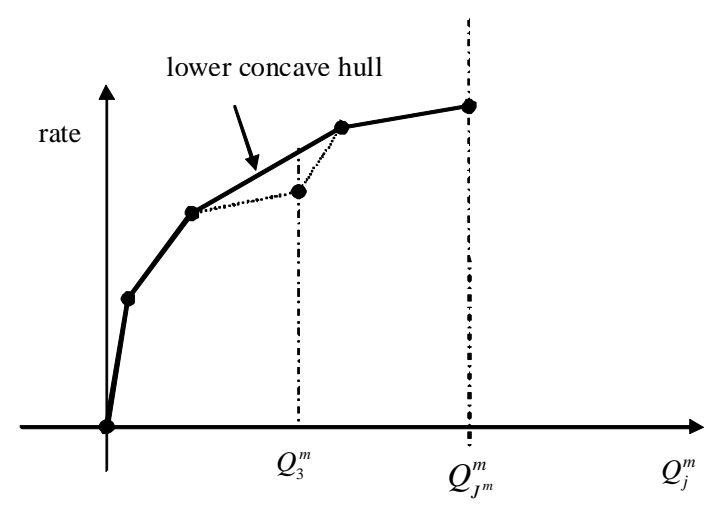

Figure 1: The rate as a function of the transmission power for some fixed state $x_{m}^{i}$. Observe that a power level which obtains a rate which is not on the lower concave hull of the interpolation ( $Q_{3}^{m}$ in the figure) would not be used: A convex combination of $Q_{2}^{m}$ and $Q_{4}^{m}$ can lead to a better rate at the same power cost.

Lemma 1. Let $\mathbf{q}=\left(\mathbf{q}^{1}, \ldots, \mathbf{q}^{M}\right)$ be a Nash equilibrium point (7). Then

$$
r^{m}(\mathbf{q})=\rho^{m}
$$

Proof. The result immediately follows from (5)-(7) by noting that both the user rate and average power consumption are monotonously increasing functions of $q_{i, j}^{m}$,

$j \in\left\{1, \ldots J^{m}\right\}, i \in\left\{1, \ldots I^{m}\right\}$. Indeed, if the current rate is strictly higher than required, each user may unilaterally reduce its power investment (and still hold to its requirement) by slightly decreasing some $q_{i, j}^{m}>0, j \in\left\{1, \ldots J^{m}\right\}$.

Noting (6), we observe that the best-response strategy can be analyzed for each user in isolation, as the overall effect of other users is manifested only through a multiplicative term which modulates the effective rate. Indeed, Lemma 1 indicates that the user's best response must obey $H^{m}\left(\mathbf{q}^{m}\right)=$ $\bar{\rho}^{m}$, where $\bar{\rho}^{m}=\frac{\rho^{m}}{\prod_{l \neq m}\left(1-p^{l}\left(\mathbf{q}^{l}\right)\right)}$. Consequently, we shall henceforth use the latter transformation for the best-response characterization.

Consider the (discrete) rate function obtained for a given state $x_{i}^{m}$, and let $f_{i}^{m}\left(Q^{m}\right)$ be its continuous interpolation. Let $g_{i}^{m}\left(Q^{m}\right)$ be the lower concave hull of $f_{i}^{m}\left(Q^{m}\right)$, i.e, the smallest concave function such that $g_{i}^{m}\left(Q^{m}\right) \geq f_{i}^{m}\left(Q^{m}\right)$ (see Figure 1). We next assert that power levels that are not on the lower concave hull $g_{i}^{m}\left(Q^{m}\right)$ would not be used in any best-response strategy.

Lemma 2. Consider any channel state $x_{i}^{m}$. Let $Q_{j}^{m}$ be a power level whose associated rate is below $g_{i}^{m}\left(Q^{m}\right)$. Then $q_{i, j}^{m}=0$ under every best-response strategy.

Proof. Assume by contradiction that $q_{i, j}^{m}>0$. Let $Q_{j_{h}}>$ $Q_{j}^{m}$ and $Q_{j_{l}}<Q_{j}^{m}$ be the two adjacent to $Q_{j}^{m}$ levels that are on the lower concave hull $g_{i}^{m}\left(Q^{m}\right)$ (see Figure 1). Then there exists some $0<\delta<1$ such that $\delta Q_{j_{h}}+(1-\delta) Q_{j_{l}}=Q_{j}^{m}$. Let $\epsilon>0$ be some small constant. Consider now the modified strategy $\tilde{\mathbf{q}}$, given by $\tilde{q}_{k, j}^{m}=q_{k, j}^{m}, k \neq i, \tilde{q}_{i, j^{\prime}}^{m}=q_{i, j^{\prime}}^{m}$, 
$j^{\prime} \notin\left\{j, j_{h}, j_{l}\right\}, \tilde{q}_{i, j}^{m}=q_{i, j}^{m}-\epsilon, \tilde{q}_{i, j_{h}}^{m}=q_{i, j_{h}}^{m}+\epsilon \delta, \tilde{q}_{i, j_{l}}^{m}=$ $q_{i, j_{l}}^{m}+\epsilon(1-\delta)$. This strategy obviously maintains the same power investment, yet obtains strictly higher rate. Hence $H^{m}\left(\tilde{\mathbf{q}}^{m}\right)>\bar{\rho}^{m}$. We may now follow the same argument as in the proof of Lemma 1 to conclude that there exist a lower-power strategy that holds to the rate requirement, in contradiction to the optimality of $\mathbf{q}$.

As a consequence of the above lemma, for any given channel state $x_{i}^{m}$, the interpolation of power levels that can be used (with the associated rates) creates a piecewise-linear concave function. Due to concavity, the next result immediately follows.

Lemma 3. Let $\mathbf{q}^{m}$ be a best-response strategy. Then for every state $x_{i}^{m}$ there are at most two non-zero elements $q_{i, j}^{m}$ and $q_{i, k}^{m}$. Moreover, these elements correspond to adjacent power-levels $Q_{j}^{m}$ and $Q_{k}^{m}$ on the lower concave hull $g_{i}^{m}$.

Proof. Fix a channel state $x_{i}^{m}$. Based on Lemma 2 we may restrict attention to power levels that are on the lower concave hull $g_{i}^{m}\left(Q^{m}\right)$. Hence, the interpolation of their corresponding rates $R_{i, j}^{m}$ is exactly $g_{i}^{m}\left(Q^{m}\right)$. To prove the claim, we next show that a strategy $\mathbf{q}$ which assigns strictly positive transmission probabilities to non-adjacent power levels (on the lower concave hull) is indeed suboptimal. Let $Q_{l}^{m}$ and $Q_{h}^{m}$ be two such power levels, and assume by contradiction that positive probabilities are assigned to these power levels. By assumption, there exists an additional power level $Q_{j *}^{m}$ (which belongs to $g_{i}^{m}\left(Q^{m}\right)$ ) such that

$$
Q_{j *}^{m}=\delta Q_{l}^{m}+(1-\delta) Q_{h}^{m},
$$

$0<\delta<1$. Consider a modified strategy $\tilde{\mathbf{q}}$ which is identical to $\mathbf{q}$ except that $\tilde{q}_{i, l}^{m}=q_{i, l}^{m}-\delta \epsilon, \tilde{q}_{i, h}^{m}=q_{i, l}^{m}-(1-\delta) \epsilon$, $\tilde{q}_{i, j *}^{m}=q_{i, j *}^{m}+\epsilon$. Noting (9), both $\tilde{\mathbf{q}}$ and $\mathbf{q}$ consume the same average power. The difference in both strategy rates, after divided by $\epsilon$ is given by $g_{i}^{m}\left(\delta Q_{l}^{m}+(1-\delta) Q_{h}^{m}\right)-$ $\left[\delta g_{i}^{m}\left(Q_{l}^{m}\right)+(1-\delta) g_{i}^{m}\left(Q_{h}^{m}\right)\right]$. This quantity is strictly positive due to concavity (and noting that $g_{i}^{m}$ is a strictly increasing function). We now repeat the same argument as in Lemma 1 to conclude that a lower-power strategy can be derived from $\tilde{\mathbf{q}}$, contradicting the optimality of $\mathbf{q}$.

The significance of Lemma 3 is that the best-response for each state can be represented by a point on the concave hull graph.

Until now we have focused on the optimal strategy within each channel state. We next wish to provide a characterization of the best-response across the different states. To that end, we require the following definition.

Definition 3.1 (RATE-GAin). Let $Q_{j}^{m}$ and $Q_{k}^{m}\left(Q_{j}^{m}<\right.$ $\left.Q_{k}^{m}\right)$ be two adjacent power-levels on the lower concave hull of $g_{i}^{m}$. The rate-gain (under state $x_{i}^{m}$ ) for these two power levels is defined as $\sigma_{i, k}^{m} \triangleq \frac{R_{i, k}^{m}-R_{i, j}^{m}}{Q_{k}^{m}-Q_{j}^{m}}$.

The next result states that higher rate-gain power levels should always be preferred. This property is central in the characterization of the best-response, and would have implications on the efficient calculation of the best-response, as well as on the equilibrium structure (See Section 3.2)
Lemma 4. Consider two channel states $x_{i}^{m}$ and $x_{i^{\prime}}^{m}$. A power allocation with $q_{i, j}^{m}<1(j \geq 1)$ and $q_{i^{\prime}, k}^{m}>0(k \geq 1)$ such that $\sigma_{i, j}^{m}>\sigma_{i^{\prime}, k}^{m}$ is always suboptimal.

Proof. The idea of the proof, is to raise the transmission probability $q_{i, j}^{m}$ and decrease $q_{i^{\prime}, k}^{m}$ while preserving the same data rate. Such strategy would lower the average power investment, and the result will follow.

Notice that according to Lemmas $2-3$ we may restrict attention to strategies in which two adjutant power levels on the lower concave hull of each state are used. To simplify notations, let $Q_{j}^{m}$ and $Q_{j-1}^{m}$ be those power levels for state $x_{i}^{m}$, and let $Q_{k}^{m}$ and $Q_{k-1}^{m}$ be those for state $x_{i^{\prime}}^{m}$. Accordingly, $\sigma_{i, j}^{m}=\frac{R_{i, j}^{m}-R_{i, j-1}^{m}}{Q_{j}^{m}-Q_{j-1}^{m}}$ and $\sigma_{i^{\prime}, k}^{m}=\frac{R_{i^{\prime}, k}^{m}-R_{i^{\prime}, k-1}^{m}}{Q_{k}^{m}-Q_{k-1}^{m}}$. For $\epsilon>0$ small, consider the modified strategy $\tilde{q}_{i, j}^{m}=q_{i, j}^{m}+\epsilon$, $\tilde{q}_{i, j-1}^{m}=q_{i, j-1}^{m}-\epsilon$. This strategy raises the average rate by $\pi_{i}^{m} \epsilon\left(R_{i, j}^{m}-R_{i, j-1}^{m}\right)$. To compensate for this rate raise, we further impose $\tilde{q}_{i^{\prime}, k}^{m}=q_{i, k}^{m}-\delta, \tilde{q}_{i^{\prime}, k-1}^{m}=q_{i, k-1}^{m}+\delta$. To equalize the rates obtained by $\mathbf{q}$ and $\tilde{\mathbf{q}}, \delta$ is chosen as $\delta=\epsilon \frac{\pi_{i}^{m}}{\pi_{i^{\prime}}^{m}} \frac{R_{i, j}^{m}-R_{i, j-1}^{m}}{R_{i^{\prime}, k}^{m}-R_{i^{\prime}, k-1}^{m}}$. The difference in power investments between $\tilde{\mathbf{q}}$ and $\mathbf{q}$ is consequently given by

$$
\begin{aligned}
& \frac{1}{\epsilon \pi_{i}^{m}}\left[\left(Q_{j}^{m}-Q_{j-1}^{m}\right)-\frac{R_{i, j}^{m}-R_{i, j-1}^{m}}{R_{i^{\prime}, k}^{m}-R_{i^{\prime}, k-1}^{m}}\left(Q_{k}^{m}-Q_{k-1}^{m}\right)\right] \\
= & \frac{1}{\left(\epsilon \pi_{i}^{m}\right)\left(R_{i^{\prime}, k}^{m}-R_{i^{\prime}, k-1}^{m}\right)}\left[\left(Q_{j}^{m}-Q_{j-1}^{m}\right)\left(R_{i^{\prime}, k}^{m}-R_{i^{\prime}, k-1}^{m}\right)\right. \\
- & \left.\left(R_{i, j}^{m}-R_{i, j-1}^{m}\right)\left(Q_{k}^{m}-Q_{k-1}^{m}\right)\right],
\end{aligned}
$$

which is strictly less than zero by the lemma's assumption.

\subsection{Equilibrium Structure and Calculation of the Best-Response}

Lemma 4 leads to several significant properties regarding the structure of the equilibrium. As expected, better channel states would be always preferred. In addition, there always exists a best-response strategy with a single randomization of power levels. These properties are summarized in the next proposition.

Proposition 1. The following properties are valid for every best-response strategy: (i) There exists some state $x_{i}^{m}$ in which the user transmits with positive probability (i.e., $0<$ $\left.\sum_{j=1}^{J^{m}} q_{i, j}^{m} \leq 1\right)$, and further $\sum_{j=1}^{J^{m}} q_{k, j}^{m}=1$ for $k>i$ and $\sum_{j=1}^{J^{m}} q_{k, j}^{m}=0$ for $k<i$. (ii) There exists a best response with a single randomization, that is for every channel state $i$ but one there exists some power level in $\mathcal{Q}^{j}$ which is used w.p. 1, i.e., $q_{i, j}^{m}=1$.

Proof. (i) Let $x_{i}^{m}$ be the lowest channel quality at which the user transmits with positive probability. Then by construction, $\sum_{j=1}^{J^{m}} q_{k, j}^{m}=0$ for $k<i$. For every $k>i$, let $Q_{j_{k}}^{m}$ be the smallest power level that is on the lower concave hull $g_{j_{k}}^{m}$. It can be easily seen that $\sigma_{k, j_{k}}^{m}>\sigma_{i, j}^{m}$ for every $Q^{j}$ that is on the lower concave hull $g_{i}^{m}$. This property essentially follows from (2). Assume by contradiction that $\sum_{j=1}^{J^{m}} q_{k, j}^{m}<1$; this means that $q_{k, j_{k}}^{m}<1$. It then follows by Lemma 4 that such strategy cannot be optimal.

(ii) In view of Lemma 4, a best-response with more than a 
single randomization is possible if and only if two (or more) rate-gains, corresponding to active power levels of two (or more) different channel states, are the same. In that case, the same interchange argument used in the proof of Lemma 4 can be applied for constructing an equal power strategy that eliminates one of the randomizations. Repeating such procedure will result in a single randomization.

REMARK 2. Classic results on "water-filling" optimization consider the specific cost function (3). For this function, it can be shown that higher powers are used for better channel states. Under our general model assumptions this is not necessarily true. Generally, an additional property of "increasing differences" is required for monotonicity in the power levels as described above. In our case, the increasing differences property holds if and only if

$$
R_{i^{\prime}, j^{\prime}}^{m}-R_{i, j^{\prime}}^{m}>R_{i^{\prime}, j}^{m}-R_{i, j}^{m}
$$

for every two indices pairs $i^{\prime}>i$ and $j^{\prime}>j$.

Based on Lemma 4, the following iterative procedure can be carried out to efficiently calculate the best-response:

1. Arrange the rate-gains in decreasing order.

2. Starting with the highest rate-gain, say $\sigma_{i, k}^{m}=\frac{R_{i, k}^{m}-R_{i, j}^{m}}{Q_{k}^{m}-Q_{j}^{m}}$, set $q_{i, k}^{m}:=1, q_{i, j}^{m}:=0$. Calculate the average rate via (5). If the total rate exceeds $\bar{\rho}^{m}$ find $q_{i, k}^{m}<1, q_{i, j}^{m}>0$ such that required rate is met with equality.

3. Otherwise, raise the rate by examining the next highest rate-gain, and setting the associated probabilities as in 2. Repeat this step until obtaining the required data rate $\bar{\rho}^{m}$.

It immediately follows from Lemma 4 and Proposition 1 that step 3 above proceeds by either augmenting the power-level for a channel state which is at use, or initiating transmissions for a new channel state (with lower quality than the ones used so far). See Figure 2 for a graphical interpretation. The above procedure guarantees convergence to the bestresponse strategy; this property easily follows from Lemma 4 .

\subsection{Power-Efficient Equilibrium}

The previous subsections established certain properties that are common to all equilibrium points. The aim of this section is to compare the possible equilibria in terms of power investment. We shall establish that the equilibrium point are ordered with respect to the individual users' power investment uniformly across all users. That is, if some user spends more power in one equilibrium than in the other, so do other users. This property immediately implies that if there exist several equilibrium points, one of them is best for all users in terms of the power investment. We shall refer to this equilibrium as the power-efficient equilibrium.

We establish below that equilibria are ordered (componentwise) in terms of the power investment. For this result, the following lemma is required.

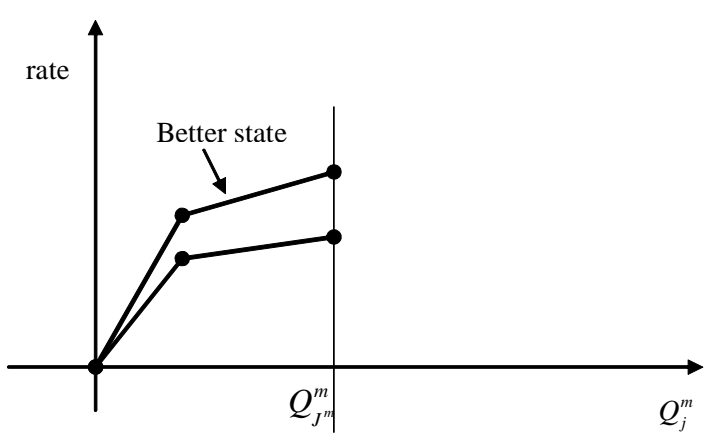

Figure 2: Consider a two-state channel with two power levels available. This graph depicts the rate as a function of the used power for both states. The procedure for optimizing the power allocation starts with using the lower power level at the best state. If the obtained rate is not satisfactory, note that in this case the preceding choice is to transmit with the lower power level also at the worse state. This example demonstrates a scenario where users may frequently transmit, resulting in frequent collisions.

Lemma 5. Assume that user $m$ is a unique network user. Consider two different rate demands $\rho^{m}$ and $\tilde{\rho}^{m}$ for that user so that $\rho^{m}<\tilde{\rho}^{m}$. Let $\mathbf{q}^{m}$ and $\tilde{\mathbf{q}}^{m}$ be best-responses for $\rho^{m}$ and $\tilde{\rho}^{m}$, respectively. Then $p^{m}\left(\mathbf{q}^{m}\right) \leq p^{m}\left(\tilde{\mathbf{q}}^{m}\right)$.

Proof. Consider the iterative procedure for obtaining the best-response strategy (see Section 3.2), which gradually raises the data rate (and the power investment) until reaching the required rate. As earlier observed, it immediately follows from Lemma 4 and Proposition 1 that each step of the above procedure proceeds by either augmenting the power-level for a channel state which is at use, or initiating transmissions for a new channel state (with lower quality than the ones used so far). Note further that a new channel state will not be used unless the transmission probability at better states equals one, hence the overall transmission probability (weakly) increases along the procedure.

The path for obtaining $\rho^{m}$ can obviously be treated as a "midway" for obtaining $\tilde{\rho}^{m}$. Consequently, based on the above observations, $p^{m}\left(\mathbf{q}^{m}\right) \leq p^{m}\left(\tilde{\mathbf{q}}^{m}\right)$ must hold.

The above lemma immediately leads to the next theorem.

THEOREM 2. Let $\mathbf{q}$ and $\tilde{\mathbf{q}}$ be two equilibria so that

$$
H^{m}\left(\mathbf{q}^{m}\right)<H^{m}\left(\tilde{\mathbf{q}}^{m}\right)
$$

for some user $m$. Then $H^{l}\left(\mathbf{q}^{l}\right)<H^{l}\left(\tilde{\mathbf{q}}^{l}\right)$ for every other user $l \neq m$. Consequently, the power investments that correspond to $\tilde{\mathbf{q}}$ are strictly higher for all users, compared to the power investments under $\mathbf{q}$.

Proof. Recall from Lemma 1 that

$$
r^{m}(\mathbf{q})=\rho^{m}
$$


for every equilibrium point q. Noting (6), dividing the equations (12) of any two users $m$ and $l$ results in the following relation $\frac{H^{m}\left(\mathbf{q}^{m}\right)\left(1-p^{l}\left(\mathbf{q}^{l}\right)\right)}{H^{l}\left(\mathbf{q}^{l}\right)\left(1-p^{m}\left(\mathbf{q}^{m}\right)\right)}=\frac{\rho^{m}}{\rho^{l}}$, or

$$
\frac{\frac{H^{m}\left(\mathbf{q}^{m}\right)}{1-p^{m}\left(\mathbf{q}^{m}\right)}}{\frac{H^{l}\left(\mathbf{q}^{l}\right)}{1-p^{l}\left(\mathbf{q}^{l}\right)}}=\frac{\rho^{m}}{\rho^{l}} .
$$

If $H^{m}\left(\mathbf{q}^{m}\right)<H^{m}\left(\tilde{\mathbf{q}}^{m}\right)$, it follows by Lemma 5 that $p^{m}\left(\mathbf{q}^{m}\right) \leq$ $p^{m}\left(\tilde{\mathbf{q}}^{m}\right)$. Hence in order to keep the fixed ratio for $\tilde{\mathbf{q}}$ it follows that $H^{l}\left(\mathbf{q}^{l}\right)<H^{l}\left(\tilde{\mathbf{q}}^{l}\right)$. It remains to verify that the power investment at $\tilde{\mathbf{q}}$ is strictly higher compared to the equilibrium q. Evidently if this was not the case, users can use $\tilde{\mathbf{q}}^{\mathbf{m}}$ as a starting point, by subtracting a small amount from some $\tilde{q}_{i, j}^{m}>0, j>1$ and obtaining a strictly lowerpower allocation for $H^{m}\left(\mathbf{q}^{m}\right)$; this contradicts $\mathbf{q}$ being an equilibrium point.

The significance of Theorem 2 is that all mobiles are betteroff at one of the equilibrium points, the power-efficient equilibrium. The next subsection is thus dedicated to finding a simple distributed mechanism which converges to this equilibrium point.

\section{BEST-RESPONSE DYNAMICS AND CON- VERGENCE TO THE POWER EFFICIENT EQUILIBRIUM}

A Nash equilibrium point for our system represents a strategically stable working point, from which no user has incentive to deviate unilaterally. Still, the question of if and how the system arrives at an equilibrium needs to be addressed. Furthermore, one of the equilibria (the power-efficient equilibrium) is best for all users, hence it is of major importance to employ mechanisms that converge to that equilibrium point. We establish below that the natural course of asynchronous best-response dynamics converges to this desirable equilibrium. Notably, these dynamics would not require specific knowledge on other user strategies, thus they can be practically applicable in wireless systems.

The distributed mechanism we consider here relies on a user's best-response, which was comprehensively studied in Section 3. Recall that in our model, the best response for user $m$ is a solution to the following optimization problem

$$
\min _{\mathbf{q}^{m} \in \Delta^{m}}\left\{\sum_{i=1}^{I^{m}} \pi_{i}^{m} \sum_{j=1}^{J^{M}} q_{i, j}^{m} Q_{j}^{m}: H^{m}\left(\mathbf{q}^{m}\right)=\bar{\rho}^{m}\right\},
$$

where $\bar{\rho}^{m}=\frac{\rho^{m}}{\prod_{l \neq m}\left(1-p^{l}\left(\mathbf{q}^{l}\right)\right)}$. Our distributed mechanism can be described as follows. Each user updates its transmission strategy from time to time through its best response (14). The update times of each user need not be coordinated with other users.

This mechanism reflects what greedy, self-interested users would naturally do: Repeatedly observe the current network conditions and react to bring their throughput to the required level, while using minimal power. For the analysis of the best-response scheme we assume the following.

\section{ASSUMPTION 1.}

(i) The user population and their throughput requirements are fixed. Users may however join the network at different times.

(ii) Each user a-priori knows its own channel state distribution.

(iii) Users repeatedly update their transmission strategies (i.e., an infinite number of updates for each user) using Eq. (14) .

(iv) The prevailing quantities $\prod_{l \neq m}\left(1-p^{l}\right)$ are perfectly estimated by the user before each update.

Our convergence result is summarized below.

Theorem 3. Assume that $\rho=\left(\rho_{1}, \ldots, \rho_{n}\right)$ admits an equilibrium point. Then under Assumption 1, the best response dynamics asymptotically converges to the power-efficient equilibrium.

The proof proceeds by showing that the vector of user transmission probabilities $\mathbf{p}$ monotonously increases until convergence. A full proof is provided in the appendix.

We briefly list here some important considerations regarding the presented mechanism.

- It is important to notice that each user is not required to be aware of the specific strategy of every other user. Indeed, only the overall idle probability of other users $\prod_{j \neq i}\left(1-p_{j}\right)$ is required in (14) (in addition to the private channel state distribution of the user which is assumed to be a-priori known). This quantity could be estimated by each user by monitoring the channel utilization.

- Assumption 1(iv) entails the notion of a quasi-static system, in which each user responses to the steady state reached after preceding user updates. This assumption approximates a natural scenario where users update their transmission probabilities at much slower time-scales than their respective transmission rates.

The assumptions used for our convergence results are obviously idealized and should be supplemented with further analysis of the effect of possible deviations from the model. Such deviations could lead the system to converge to worse equilibria, which is obviously undesirable. If such behavior is detected, users can reset their probabilities and restart the best-response mechanism for converging to the powerefficient equilibrium. This procedure resembles the basic ideas behind TCP protocols. The exact schemes for detecting suboptimal network operation, and consequently directing the network to the best equilibrium, are beyond the scope of the present paper.

\section{EQUILIBRIUM (IN)EFFICIENCY AND BRAESS-LIKE PARADOXES}

In the previous two section we have indicated that there exists a best equilibrium in terms of power investment. Furthermore, this equilibrium can be reached through bestresponse dynamics. Our first objective in this section is to 
examine whether the best equilibrium is also socially optimal. In [13] it was shown that this is the case when a single power level is available to each user. We next demonstrate by means of an example that this property generally does not carry over to our model. We then investigate the consequences of providing users with multiple power levels, and relate our observations to the well-known Braess paradox.

Recently, there has been much work in quantifying the "efficiency loss" incurred by the selfish behavior of users in networked systems (see [15] for a comprehensive review). The two concepts which are most commonly used in this context are the price of anarchy (PoA), which is (an upper bound on) the performance ratio (in terms of a relevant social performance measure) between the global optimum and the worst Nash equilibrium, and price of stability (PoS), which is (an upper bound on) the performance ratio between the global optimum and the best Nash equilibrium.

Returning to our specific network scenario, consider the case where a central authority, which has full information regarding the channel state distributions of every user can enforce a stationary transmission strategy (see Definition 2.1) for every user $m \in \mathcal{M}$. We consider the total power consumption as the system-wide performance criterion, namely

$$
C(\mathbf{q}) \triangleq \sum_{m \in \mathcal{M}} \sum_{i=1}^{I^{m}} \pi_{i}^{m} \sum_{j=1}^{J^{M}} q_{i, j}^{m} Q_{j}^{m} .
$$

Our aim is to compare the performance of the optimal centrally assigned multi-strategy to the performance at the Nash equilibrium with respect to the quantity $C(\mathbf{q})$.

A simple example in [13] shows that the PoA generally unbounded. The example obviously holds for our (more general) model as well. The fact that the power-efficient equilibrium can be reached by a distributed asynchronous mechanism (as established in Theorem 3), makes the price of stability more significant, as the price of anarchy can be avoided by employing the mechanism.

We next show through a numeric example that unlike the single power case, there can be a gap between the powerefficient equilibrium and the optimal solution.

Example 1. We consider a wireless network of two symmetric users, with identical channel conditions and rate requirements $\rho^{1}=\rho^{2}=\rho$ (hence user indexes are omitted in the sequel). The rate per (state,power) pair is given by (3). The possible channel states are $\mathcal{X}=\{0.1,0.5,5,30,80,200\}$ and the corresponding steady state distribution is

$$
\pi=(0.3,0.25,0.2,0.12,0.08,0.05) .
$$

We consider two different game instances:

- Instance 1: Multiple power levels are allowed for each user; $\mathcal{Q}^{(1)}=\{0,1,2,3,6,8,15\}$.

- Instance 2: A single power level (besides zero) is allowed; $\mathcal{Q}^{(2)}=\{0,2\}$.

Figure 3 depicts the per-user energy at equilibrium as a function of the required data rate $\rho$ (note that multiple equilibria

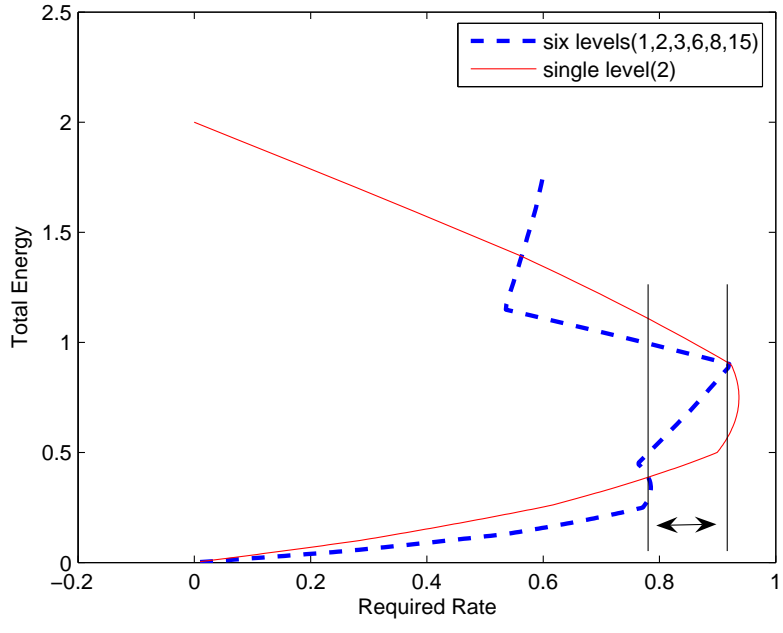

Figure 3: The average energy as a function of the required rate. Note that multiple equilibria are possible for a given $\rho$. In the marked region of required rates, the power-efficient equilibrium with a single power level outperforms the respective one with multiple power levels.

are possible for a given $\rho$ ).

The interesting region of required rates is $\rho \in[0.78,0.92]$ (emphasized in the figure itself). For rates at this region, the power-efficient equilibrium of Instance 2 (a single power level) obtains lower energy investment compared to the powerefficient equilibrium of Instance 1 (multiple power levels). Noting that the single power level of 2 is one of the available powers in $\mathcal{Q}^{(1)}$ indicated that the power-efficient equilibrium for the multiple-power case is not a system-wide optimal multi-strategy. Indeed, a simple power strategy that uses a single power only outperforms the equilibrium of Instance 1. Hence, the optimal centrally assigned strategy would obviously outperform the equilibrium policy of Instance 1 as well.

We have demonstrated through an example that the price of stability is generally larger than 1 . A precise quantification for this measure obviously depends on the channel characteristics, the available powers and the assumptions on the rate functions. Explicit models and their associated price of stability are an interesting subject for future research.

A classic example for the consequences of self-interested behavior in networks is the Braess paradox [16], which shows that the addition of a link in a transportation network might increase the overall traffic delay at equilibrium. We next point to Braess like paradoxes in our network model, which concern the addition of available power levels to each user.

The first Braess-like paradox has already been demonstrated in Example 1 of the previous section. Recall that for required rates of $\rho \in[0.78,0.92]$, Instance 2 (a single power level) outperforms Instance 1 (multiple power levels, which include the one in Instance 2). Apparently, the addition 


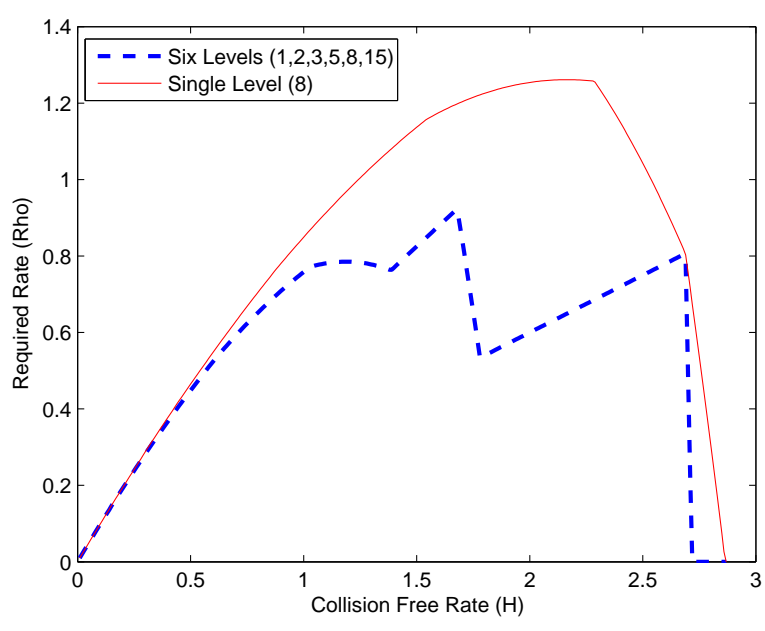

Figure 4: The obtained rate $\rho$ as a function of the collision free rate $H$. Note that several equilibrium points (up to six in this particular case) are possible for each $\rho$. It is clearly seen that the use of a single power level may accommodate larger $\rho$ 's, compared to the case where multiple power levels are used.

of power levels worsens user performance in terms of their average energy investment.

We next demonstrate an additional type of Braess-like paradox, which relates to the network's capacity. We use the term "capacity" for the (total) maximal rate that can be obtained in the network. Consider the following example.

Example 2. As in Example 1, we compare two scenarios that differ in the allowed power levels. All conditions are identical to the ones in Example 1. The only difference is that instead of Instance 2 we consider the following instance:

- Instance 3: A single power level (besides zero) is allowed; $\mathcal{Q}^{(3)}=\{0,8\}$. Note that $\mathcal{Q}^{(3)} \subset \mathcal{Q}^{(1)}$;

Figure 4 presents the throughput $\rho$ that is obtained as a function of the collision-free data rate $H^{m}(5)$. Observe that the use of a single power level increases the maximal rate $\rho$ that can be accommodated in the network. This example indicates that the use of multiple power levels might decrease the network capacity, due to selfish user behavior.

\section{DISCUSSION}

The explanation for the Braess-like paradoxes, as well as for the sub-optimality of the power-efficient equilibrium is quite intuitive, given the nature of the collision channel. In some cases, user strategies would result in frequent transmissions; this would be the case if the rate-gain that is obtained at low power levels, even at inferior channel states, is higher than the rate-gain while switching to higher power levels. Figure 2 illustrates this idea. Consequently, the shared channel would be subject to frequent collisions that would lead to both unnecessary power investment and a decrease in network capacity.
It is well known that the adjustment of power levels increases capacity in single-user channels [5]. A challenging direction for future research, inspired by the above observations, is how to prevent the Braess-like paradoxes in multiuser wireless networks, and even better, enlarge capacity and reduce power investment. A key role can be given to network management that should determine the right tradeoff between user flexibility and overall performance, by assigning the power levels appropriately.

\section{CONCLUSION}

This paper has studied the non-cooperative game over a shared collision channel, where mobiles adjust their transmission power based on local channel information. Mobiles are heterogenous in terms of both their physical parameters (channel distribution, available powers) and QoS parameters (the required rates). We have characterized the best response water-filling-like strategy, and consequently showed that one equilibrium point is best for all users in terms of power consumption. However, we showed that the best equilibrium is generally suboptimal; moreover, phenomena similar to the Braess paradox might occur when additional power levels are made available to the users. This factor should be considered in the design and management of wireless systems.

The framework and results of this paper may be extended in several ways. A central issue in any specific model would be how to detect suboptimal equilibria and lead the network to the best equilibrium point. A related direction is to examine the resilience of best-response mechanisms, such as the one suggested here, to changes in the transmitters population, which obviously occur in wireless networks. An additional research direction is to extend the reception model beyond the collision model studied in this paper. In particular, capture models (which sometimes better represent WLAN systems) are of obvious interest. In these models, the use of higher power levels increases not only the data rate, but also the chances of the individual data for being properly received at the base station. Hence, selfish mobiles have a natural incentive to transmit at high power, which is usually not the case for the collision model studied here. It will be therefore interesting to examine whether some of the negative consequences of selfish behavior reported here disappear, thereby reducing the gap between the power-efficient operating point and the optimal one.

\section{ADDITIONAL AUTHORS}

\section{REFERENCES}

[1] E. Biglieri, J. Proakis, and S. Shamai, "Fading channels: Information-theoretic and communications aspects," IEEE Transactions on Information Theory, vol. 44, no. 6, pp. 2619-2692, 1998.

[2] F. Meshkati, H. V. Poor, and S. C. Schwartz, "Energy-efficient resource allocation in wireless networks," IEEE Signal Processing Magazine, vol. 24, no. 3, 2007.

[3] X. Qin and R. Berry, "Distributed approaches for exploiting multiuser diversity in wireless networks," IEEE Transactions on information theory, vol. 52, no. 2, pp. 392-413, 2006. 
[4] S. Adireddy and L. Tong, "Exploiting decentralized channel state information for random access," IEEE Transactions on Information Theory, vol. 51, no. 2, pp. 537-561, 2005.

[5] A. Goldsmith and P. P. Varaiya, "Capacity of fading channels with channel side information," IEEE Transactions on Information Theory, vol. 43, no. 6, 1997.

[6] "IEEE 802.11 standards."

[7] D. Bertsekas and R. Gallager, Data Networks. Prentice-Hall, 1992.

[8] C. Saraydar, N. N.B. Mandayam, and D. Goodman, "Efficient power control via pricing in wireless data networks," IEEE Transactions on Communications, vol. 50, no. 2, pp. 291-303, 2002.

[9] E. Altman and Z. Altman, "S-modular games and power control in wireless networks," IEEE Transactions on Automatic Control, vol. 48, no. 5, pp. 839-842, 2003.

[10] T. Alpcan, T. Başar, R. Srikant, and E. Altman, "CDMA uplink power control as a noncooperative game," Wireless Networks, vol. 8, pp. 659-670, 2002.

[11] L. Lai and H. El-Gamal, "The water-filling game in fading multiple access channel," IEEE Transactions on Information Theory, 2006, submitted.

[12] E. Altman, K. Avrachenkov, G. Miller, and B. Prabhu, "Discrete power control: Cooperative and non-cooperative optimization," in INFOCOM, 2007.

[13] I. Menache and N. Shimkin, "Efficient rate-constrained Nash equilibrium in collision channels with state information," EE Department, Technion, Tech. Rep. CCIT No. 626, July 2007, available from http://www.ee.technion.ac.il/people/shimkin/ PREPRINTS/CSI_Collision07full.pdf.

[14] D. Fudenberg and J. Tirole, Game Theory. MIT Press, 1991

[15] T. Roughgarden, Selfish Routing and the Price of Anarchy. MIT Press, 2005.

[16] D. Braess, "Uber ein paradoxon aus der verkehrsplanung," Unternehmensforschung, vol. 12, pp. $258-268,1969$.

\section{APPENDIX}

\section{A. PROOF FOR THEOREM 3}

For the proof, we use the following notations. Let the update time-slots of each user $m$ be given by an increasing sequence $\left\{t_{k}^{m}\right\}, k \in\{1,2,3, \ldots\}$. Also, let

$$
\left\{t_{k}\right\}=\left\{\left\{t_{k}^{1}\right\} \cup\left\{t_{k}^{2}\right\} \cup \ldots\left\{t_{k}^{M}\right\}\right\},
$$

$k \in\{1,2, \ldots\}$. Note that at each $t_{k}$ at least a single user updates its transmission probability. We shall use the notation $p_{k}^{m}$ for the transmission probability of user $m$ at time $t_{k}$ (similarly, $\mathbf{p}_{k}$ is the transmission probability vector at time $t_{k}$ across all users, and $\mathbf{p}_{k}^{-m}$ is the vector of all users but the $m$ th one), with the convention of $p_{0}^{m}=0$ for every user $m$. With some abuse of notation, we use the notation $r^{m}\left(H^{m}, \mathbf{p}^{-m}\right)$ for the user's rate, as determined by its collision-free rate $H^{m}(5)$ and the transmission probabilities of other users. Subscripts for these quantities will denote the time epoch. The explicit dependence of these quantities in $\mathbf{q}$ is not denoted, for simplicity of the exposition.
For the proof of the theorem we require the next lemma.

\section{LEMMA 6. The sequence $\mathbf{p}_{k}$ is increasing.}

Proof. The result follows by induction. Obviously, $\mathbf{0}=$ $\mathbf{p}_{0} \leq \mathbf{p}_{1}$, as at least a single user accesses the channel at $k=1$. Assume that $\mathbf{p}_{0} \leq \mathbf{p}_{1} \leq \ldots \mathbf{p}_{k-1}$. We next show that $\mathbf{p}_{k-1} \leq \mathbf{p}_{k}$. Denote by $M_{k}$ the set of users who update their probabilities at time $k$ (so that $p_{k-1}^{m}=p_{k}^{m} \forall m \notin M_{k}$ ). For each $m \in M_{k}$, let $k^{m}<k$ be the last time epoch at which user $m$ updated its probability. Note that

$$
r^{m}\left(p_{k-1}^{m}, \mathbf{p}_{k^{m}-1}^{-m}\right)=r^{m}\left(p_{k}^{m}, \mathbf{p}_{k-1}^{-m}\right)=\rho^{m},
$$

by Lemma 1 .

Since $r^{m}$ decreases in $\mathbf{p}^{-m}$ and, by assumption, $\mathbf{p}_{k_{i}-1}^{-m} \leq$ $\mathbf{p}_{k-1}^{-m}$, it follows that $H_{k-1}^{m} \leq H_{k}^{m}$ (as $r_{i}$ is increasing in $\left.H^{m}\right)$. Since both these quantities are obtained through bestresponses, it follows from Lemma 5 that $p_{k-1}^{m} \leq p_{k}^{m}$

It follows from the above lemma that either some component of $\mathbf{p}$ must exceed 1 at some iteration, or else $\mathbf{p}$ approaches a limit, say $\mathbf{p}^{*}$, and in this limit the equations (8) are obviously satisfied (by continuity), i.e., it is an equilibrium point.

To conclude the proof, we now turn to show that if $\tilde{\mathbf{p}}$ is (another) equilibrium point, then $\mathbf{p}^{*} \leq \tilde{\mathbf{p}}$, hence $\mathbf{p}^{*}$ is the power efficient equilibrium (by the monotonicity of $p^{m}$ in $H^{m}$ and the monotonicity of $H^{m}$ in the total power investment). To see this, we apply a similar induction as that of Lemma 6, and also use the notations thereof. Obviously $\mathbf{0}=\mathbf{p}_{0} \leq \tilde{\mathbf{p}}$. Assume $\mathbf{p}_{0} \leq \mathbf{p}_{1} \leq \cdots \leq \mathbf{p}_{k-1} \leq \tilde{\mathbf{p}}$. Noting that $r^{m}\left(H_{k}^{m}, \mathbf{p}_{k^{m}-1}^{-m}\right)=r^{m}\left(\tilde{H}^{m}, \tilde{\mathbf{p}}^{-m}\right)=\rho^{m}$ and $\mathbf{p}_{k m-1}^{-m} \leq \tilde{\mathbf{p}}^{-m}$ for every $m \in \mathcal{M}$, it follows that $H_{k}^{m} \leq \tilde{H}^{m}$, hence $\mathbf{p}_{k} \leq \tilde{\mathbf{p}}$. This argument also shows that if some component of $\mathbf{p}_{k}$ exceeds 1 for some $k$, then there is no equilibrium point (i.e., the set of required user rates $\left\{\rho^{m}\right\}$ is infeasible). 